\title{
Morphofunctional Significance of "In Vivo Cryotechnique" for Analyses of Cells and Tissues in Living Animal Organs
}

\author{
S. Ohno, N. Terada, N. Ohno, S. Saitoh, Y. Saitoh and Y. Fujii
}

Department of Anatomy and Molecular Histology, Interdisciplinary Graduate School of Medicine and Engineering, University of Yamanashi, Chuo-city, Yamanashi 409-3898, Japan

Morphological study has been one of the major approaches to understand physiological and pathological features of living animal organs. For morphological observation, chemical fixation and alcohol dehydration have been commonly used as easy preparation procedures, but they bring about morphological artifacts of dynamically changing tissues in vivo (Fig. 1a,b). To the contrary, both quick-freezing and high-pressure freezing can be contributed to reduction of such artifacts (Fig. 1c,d), but specimens have to be resected from living animal organs, inevitably resulting in ischemia and anoxia. We already developed "in vivo cryotechnique" (IVCT) to clarify morphofunctional features of living animal organs (Fig. 1f) [1], and reported dynamically changing morphology (Fig. 2) and immunolocalization of functional proteins [2]. The purpose of this study is to describe technical procedures and significance of IVCT [3] and report some findings obtained by IVCT.

The IVCT was an original technique to cryofix living animal organs without separating their tissues from blood circulation (Fig. 1f) [1]. After exposing living animal organs under anesthesia, it is quickly performed by cutting them with a cryoknife precooled in liquid nitrogen $\left(-196^{\circ} \mathrm{C}\right)$ and simultaneously pouring isopentane-propane (IP) cryogen $\left(-193^{\circ} \mathrm{C}\right)$ over them under "in vivo cryoapparatus" (Eiko Corporation, Ibaraki, Japan) [2]. The operation manual was already described in another paper [2]. By combination of the cryoknife with IP cryogen, vitrification of frozen tissues can be obtained within several micrometers from cryocut tissue surface at an electron microscopic level (Fig. 2). Additionally, at a light microscopic level, tissue areas without detectable ice crystals are deeper than a few hundreds of micrometers, because of lower resolution of light microscopy $[4,5]$. The most significant point of IVCT is preservation of blood circulation at the exact moment of freezing [6]. Then, various following steps are chosen for each morphological analysis (Fig. 1e,h,i). Some extraction of lipid-soluble components might be induced in organic solvents during freezesubstitution (Fig.1e). But, by replica electorn microscopy (Fig. 1h), replica membranes usually retain all components of cells and tissues (Fig. 3a) [7,8]. While replicating freeze-fractured tissues with platinum and carbon, they are supposed to hold deeply etched molecules. Therefore, additional immunostaining procedure enables us to visualize them on the replica membranes. We reported replica immunolocalization of caveolin in smooth muscle cells of living mouse intestines (Fig. 3b) [7]. Moreover, replica immunoelectron microscopy was also applied to extracellular localizations of hyaluronic acid and fibronectin in rat mandibular condylar cartilage [8]. Therefore, the combination of IVCT with replica immunostaining enables us to examine the most native intra- or extracellular distribution of functional molecules in cells and tissues.

Our ultimate goal is to obtain the real morphology of living animal organs and also immunohistochemical localization of their functional molecules [3]. The IVCT is a first step for keeping blood circulation of living animals (Fig. 1f), which can be followed with various microscopic procedures (Fig. 1), resulting in the field of functional morphology closer to their living states [6]. 
References

[1] S. Ohno et al., Virchows Arch. 427 (1996) 519.

[2] N. Ohno et al., Biomed. Rev. 15 (2004) 1.

[3] S. Ohno et al., J. Electron Microsc. 59 (2010) 395.

[4] N. Terada et al., J. Histochem. Cytochem. 57 (2009) 883.

[5] S. Shimo et al., J. Immunol. Methods 361 (2010) 64.

[6] N. Terada et al., Microvasc. Res. 80 (2010) 491.

[7] I. Takayama et al., Histochem. Cell Biol. 112 (1999) 443.

[8] Z. Zea-Aragon et al., Histochem. Cell Biol. 121 (2004) 255.

[9] The Seto Prize 2007 was awarded to S.O. for his "Development of In Vivo Cryotechnigue" by The Japanese Society of Microscopy.
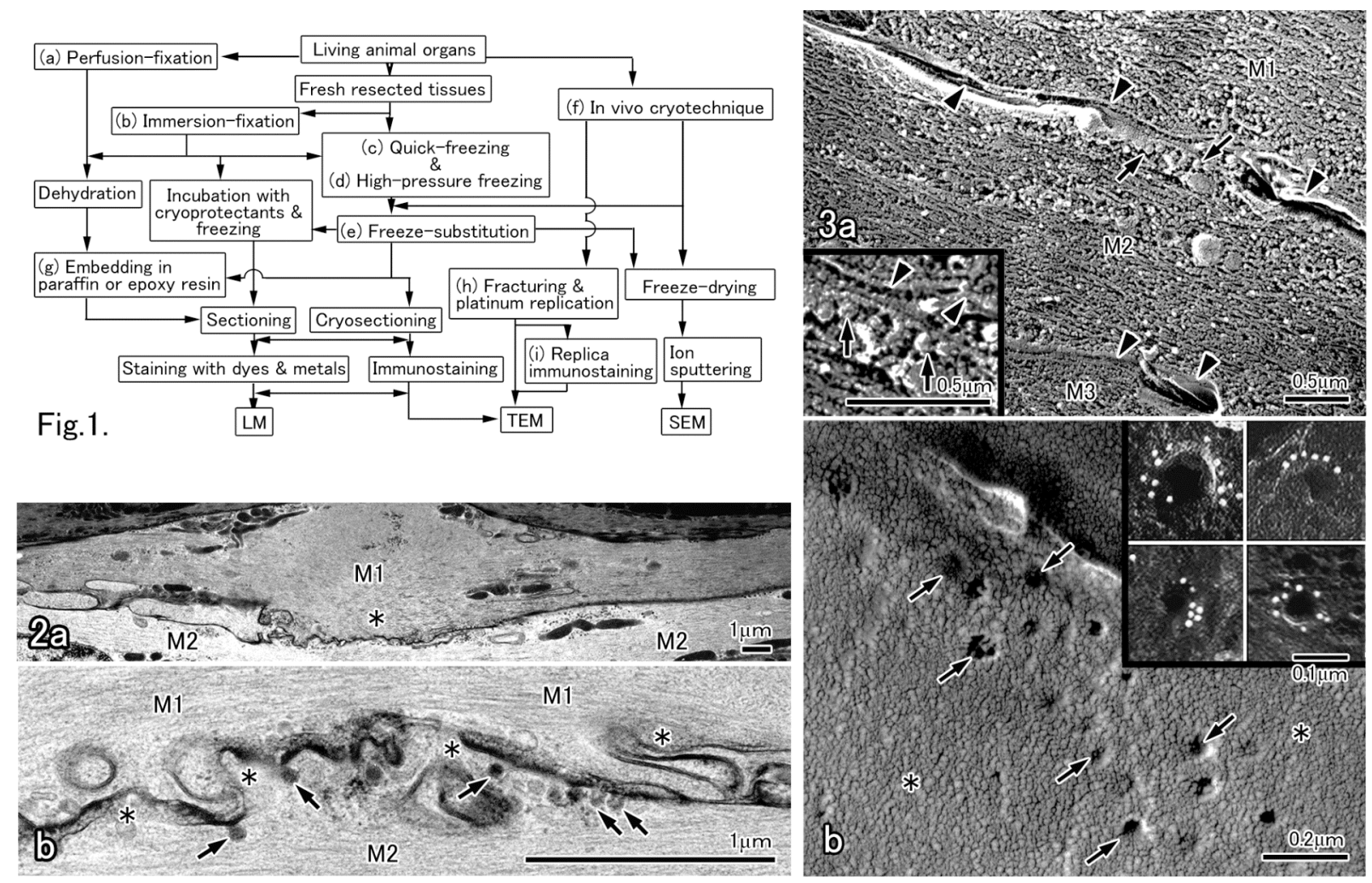

FIG. 1. Flowchart of preparation steps for LM, TEM and SEM. Perfusion- (a) and immersionfixation (b), quick-freezing (c) and high-pressure freezing (d) and IVCT (f) in connection with their following preparation steps.

FIG. 2. Electron micrographs of intestinal smooth muscle cells in living mice prepared by the IVCT. (a) Outer layers of smooth muscle cells (M1 and M2) show interdigitation of sarcolemma (asterisk). (b) Contracting muscle cells (M1 and M2) are tightly attached to each other (asterisks). Arrows; caveolae.

FIG. 3. Replica micrographs of intestinal muscle cells prepared by IVCT, followed by replica preparation. (a) Freeze-fractured muscle cells (M1-M3) show cytoskeletons and caveolar structures (arrows) along sarcolemma (arrowheads). (b) Various types of caveolar neck parts (arrows) are seen on freeze-fractured P-face (asterisks). Inset; immunostaining for caveolin with immunogold method. 